\title{
The Star Formation History of Damped Lyman-Alpha Systems
}

\author{
Arthur M. Wolfe \\ University of California, San Diego, CASS, 9500 Gilman Drive, La \\ Jolla, CA, 92093-0424
}

\begin{abstract}
The $\mathrm{C} \mathrm{II} *$ technique for measuring star formation rates (SFRs) in damped Ly $\alpha$ systems (DLAs) is described. We measure cooling rates of the gas from the strength of $\mathrm{C} \mathrm{II}^{*} 1335.7$ absorption lines, and infer heating rates by assuming the gas is heated by the grain photoelectric mechanism. Since the heating rates depend on the intensity of FUV radiation, we deduce the SFRs per unit area, which are comparable to the value in the Milky Way disk. From DLA statistics we obtain the SFRs per unit comoving volume at $z=[1.6,4.5]$, which are similar to the rates in Lyman Break Galaxies. Implications such as the presence of a two-phase medium, metal production, and feedback processes are described. The model is tested and found to be consistent with observations.
\end{abstract}

\section{Introduction}

In this talk I describe a new technique for measuring SFRs in protogalaxies. The technique differs from traditional methods, which rely on the detection of starlight (e.g. Steidel et al. 1999). Rather, it relies on feedback between radiation emitted by newly formed stars and the surrounding interstellar gas. Specifically, we detect the gas in absorption against background QSOs in the C II* 1335.7 transition, which measures the rate at which the gas is heated. Therefore, the sample of protogalaxies is cross-section weighted rather than luminosity weighted. As a result the SFRs we obtain are not limited to the most luminous objects, but instead refer to a more representative portion of the protogalactic mass distribution.

Our sample of protogalaxies is comprised of DLAs, a population of high- $z$ layers of neutral gas, which dominate the neutral gas content of the universe at $z=[0,5]$, and contain sufficient gas to account for a significant fraction of visible stars in modern galaxies (e.g. Storrie-Lombardi \& Wolfe 2000). For these reasons the DLAs are widely regarded to be the progenitors of modern galaxies (Peebles 1993).

\section{The $\mathrm{C} \mathrm{II}^{*}$ Technique}

Assume neutral gas in DLAs is heated by the same grain photoelectric mechanism that heats the ISM (Bakes \& Tielens 1994). In that case the heating rate per $\mathrm{H}$ atom is given by $\Gamma_{d}=10^{-24} \kappa \epsilon G_{0} \mathrm{erg} \mathrm{s}^{-1} \mathrm{H}^{-1}$, where $\kappa$ is the dust-to-gas ratio relative to the Milky ISM, $\epsilon$ is the grain photoelectric heating efficiency, and $G_{0}=4 \pi J / 1.6 \times 10^{-2} \mathrm{ergs} \mathrm{cm}^{-2} \mathrm{~s}^{-1}$ where $J$ is the FUV mean intensity (Bakes \& 


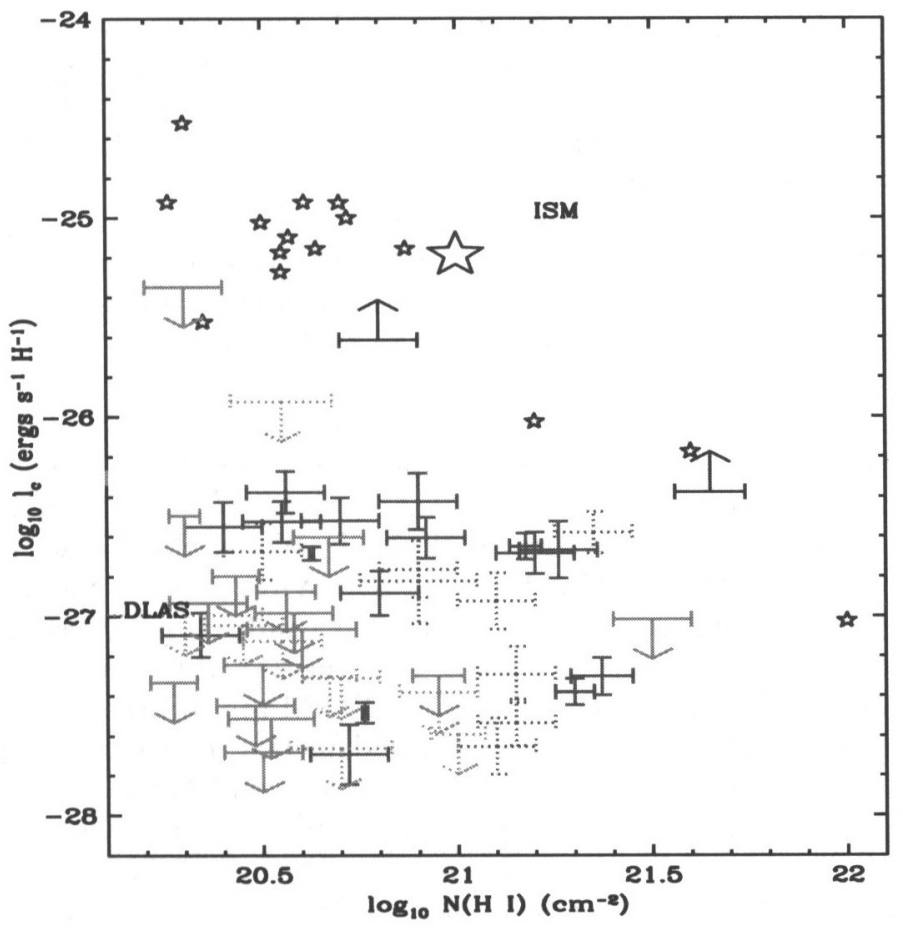

Figure 1. $\quad l_{c}$ versus $N(\mathrm{H} \mathrm{I})$. Data points with error bars show positive detections, and $2-\sigma$ upper and lower limits. Solid and dashed data points correspond to HIRES and ESI data respectively. Small stars depict detections toward H I clouds in the ISM. The large star is the spontaneous emission rate per $\mathrm{H}$ atom averaged over the ISM

Tielens 1994). Because of their low dust content, plane parallel layers comprising DLAs are optically thin to FUV radiation. Therefore, $J$ is proportional to the line integral $\int \rho_{L} d s$ where the source function $\rho_{L}$ is the luminosity density of starlight in DLAs. Thus, $J$ is proportional to the projected luminosity per unit area, which is proportional to $\dot{\psi}_{*}$ since FUV radiation comes from short-lived upper main-sequence stars. Consequently, a measurement of $J$ determines $\dot{\psi}_{*}$. Because $\epsilon$ has been calculated (Bakes \& Tielens 1994) and it is straightforward to determine $\kappa$, we can obtain $\dot{\psi}_{*}$ provided we measure the heating rate.

We determine the heating rate by equating it to the cooling rate. In the ISM, cooling is dominated by [C II] $158 \mu \mathrm{m}$ emission, which results from the transition between the ${ }^{2} P_{3 / 2}$ and ${ }^{2} P_{1 / 2}$ fine-structure states. Let the density-weighted cooling rate averaged along the line-of-sight be given by $l_{c}=N\left(\mathrm{CII}^{*}\right) h \nu_{u l} A_{u l}$ $/ N(\mathrm{HI})$ erg s $\mathrm{s}^{-1} \mathrm{H}^{-1}$ (Pottasch 1979) where $h \nu_{u l}$ and $A_{u l}$ are the energy and spontaneous emission coefficient for the fine-structure transition, and $N\left(\mathrm{C} \mathrm{II}{ }^{*}\right)$ is the column density of $\mathrm{C}^{+}$ions in the ${ }^{2} P_{3 / 2}$ state. We obtain $l_{c}$ from $\mathrm{UV}$ spectroscopy by inferring $N(\mathrm{C} \mathrm{II})$, from $\mathrm{C} \mathrm{II}^{*} 1335.7$ absorption lines arising 
from the ${ }^{2} P_{3 / 2} \rightarrow{ }^{2} D_{3 / 2}$ transition and $N(\mathrm{H} \mathrm{I})$ from the damped Ly $\alpha$ line. Using the HIRES and ESI spectrographs on the Keck $10 \mathrm{~m}$ telescopes we have determined $l_{c}$ for 50 DLAs. The results shown in Figure 1 indicate that $l_{c}$ for the main body of DLA detections is about $1 / 30$ of $l_{c}$ for the ISM. The ratio of the two heating rates is simply explained if the DLA and ISM gas are heated by the same grain photoelectric effect. Moreover, if $G_{0}$ and $\epsilon$ in DLAs assume the same values as in the ISM, the ratio of the heating rates equals the ratio of the dust-to-gas ratios, $\kappa$, which according to Pettini et al. (1994) is remarkably close to $1 / 30$. This suggests that $\dot{\psi}_{*}$ in DLAs is similar to that in the ISM and that the DLA heating rates are lower only because of the paucity of dust.

We checked this hypothesis by computing the thermal equilibria of gas heated by cosmic rays, X-rays, and FUV radiation, and which cools via excitation of fine-structure transitions of abundant low ions, Ly $\alpha$ radiation, and grain radiative recombination (see Wolfire et al. 1995; Wolfe, Prochaska, \& Gawiser 2003; hereafter WPG): we assumed the ratio of X-ray and cosmic ray heating rates to $\dot{\psi}_{*}$ to be the same as in the ISM. The resulting equilibria admit two thermally stable phases: a warm neutral medium (WNM) and a cold neutral medium (CNM). By assuming the CNM and WNM are in pressure equilibrium at a unique pressure, $P_{e q}$, we infer the stable densities, $n_{C N M}$ and $n_{W N M}$, and quantities such as temperature, ionization fraction, and $l_{c}$ for each phase. We find that while [C II] $158 \mu \mathrm{m}$ emission is the dominant coolant in the CNM, it is only a small fraction of the total cooling rate in the WNM.

For these reasons we considered two models in which CNM and WNM gas are in pressure equilibrium at $P=P_{e q}$ : a CNM model in which the QSO sightline intersects comparable column densities of CNM and WNM gas, and a WNM model in which only the WNM gas is encountered. We constructed 2-phase models for each of the DLAs in our sample. For a DLA with a given metallicity, $[\mathrm{M} / \mathrm{H}]$, and $\kappa$ we solve the transfer equation for $G_{0}$ corresponding to a selected $\dot{\psi}_{*}$ for a uniform disk and vary $\dot{\psi}_{*}$ until the predicted $l_{c}$ at $n_{C N M}$ or $n_{W N M}$ agrees with the observed $l_{c}$ (see WPG for details). The results show no evidence for evolution of $\dot{\psi}_{*}$ in either model for $z=[1.6,4.5]$. Furthermore, the mean SFR per unit area, $\left\langle\dot{\psi}_{*}\right\rangle$, is comparable to the Milky Way rate for the CNM model, but, as expected, is much higher for the WNM model.

\section{Global SFRs and Implications}

We deduce the SFR per unit comoving volume, $\dot{\rho}_{*}(z)$, a quantity with cosmological significance, by combining $\left\langle\dot{\psi}_{*}(z)>\right.$ for a given redshift bin with an expression for the incidence of DLAs per unit absorption distance, $d \mathcal{N} / d X$. The redshift dependence of $\dot{\rho}_{*}(z)$ implies a luminosity density history that gives rise to background radiation. The bolometric background intensity generated by the WNM model exceeds the $95 \%$ confidence upper limits placed on the background intensity (e.g. Hauser \& Dwek 2001) and is therefore ruled out (Wolfe, Gawiser, \& Prochaska 2003; hereafter WGP). The resulting $\dot{\rho}_{*}(z)$ for the surviving CNM model are shown in Figure 2. The approximate agreement between $\dot{\rho}_{*}(z)$ determined for DLAs and Lyman Break Galaxies (LBGs) is either a coincidence 


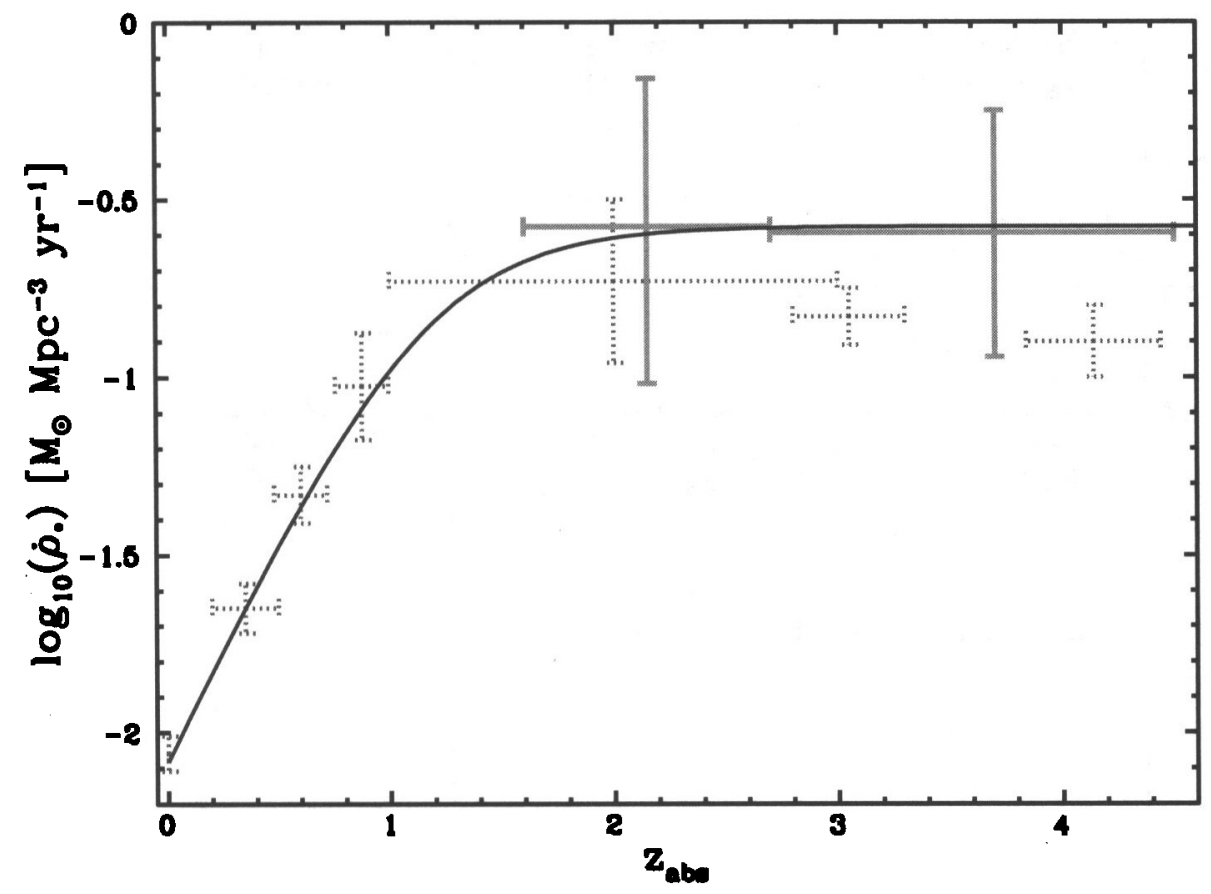

Figure 2. Solid data points depict $\dot{\rho}_{*}$ and $68 \%$ confidence errors for consensus CNM model. Dotted data points are from galaxies detected in emission: two highest redshift points are for LBGs (see WPG). Smooth curve is eyeball fit to consensus CNM model and galaxy data. Cosmology with $\Omega_{\mathrm{M}}=1.0, \Omega_{\Lambda}=0.0$, and $h=0.5$ assumed. Note, the DLA data points include systematic errors not included in the galaxy data.

or indicates a connection between DLAs and LBGs. This question is currently under investigation.

Our results have several implications (see WGP). First, time integrals of the $\dot{\rho_{*}}(z)$ curve in Figure 2 result in the densities of stars and metals at a given redshift. Although the mass of stars produced by $z=0$ is consistent with the masses of stellar populations in modern galaxies, the mass of metals produced by $z=2.5$ is more than a factor of 20 higher than is observed in DLAs at that redshift. This generic problem also appears in CDM models (Nagamine et al. 2003) and in semi-analytic models (e.g. Somerville et al. 2001), but is absent in the models of Pei et al. (1999), which account for selection biases due to dust. We suggest that star formation confined to a centrally located "bulge" region offers a possible solution to this dilemma. In this scenario, rapid metal enrichment occurs in a compact bulge, an old but metal-rich population (Wyse et al. 1997), while FUV radiation leaks out of the bulge to heat the spatially extended H I gas detected in absorption. WGP find that the $\dot{\rho}_{*}(z)$ predicted for the bulge scenario is the same as for star formation throughout a uniform disk. 


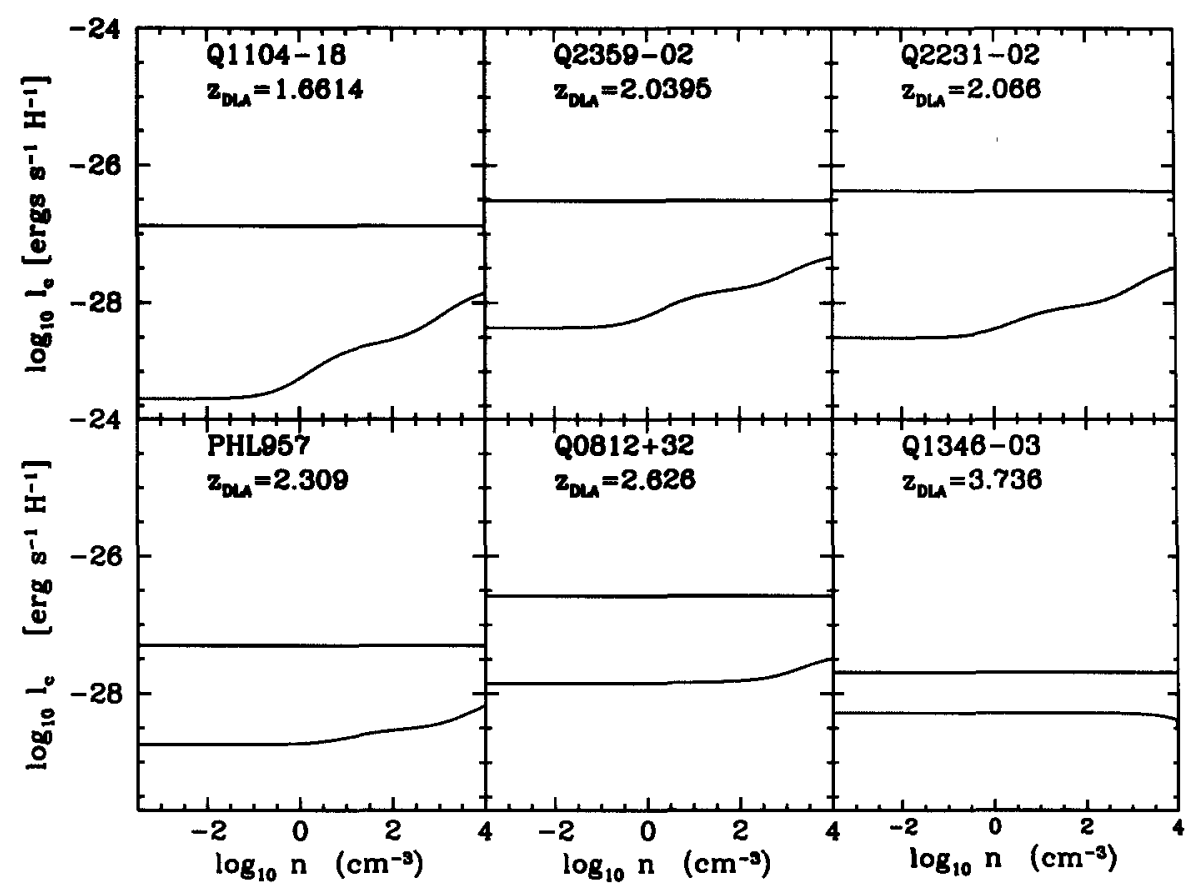

Figure 3. $\quad l_{c}$ versus density for 6 DLAs heated by FUV background radiation alone. CMB excitation dominates at low $n$, grain photoelectric heating dominates at intermediate $n$, and $\mathrm{C} \mathrm{I}$ photoionization heating dominates at highest $n$. Measured $l_{c}$, depicted as horizontal lines, have typical 1- $\sigma$ error bars of \pm 0.2 dex.

Second, WGP searched for evidence of feedback and found tentative evidence in two cases. They found evidence, at $2-\sigma$ significance, for a correlation between $\dot{\psi}_{*}$ and $[\mathrm{Si} / \mathrm{H}]$, which, if confirmed, would be consistent with similar correlations found in nearby galaxies (Garnett et al. 1997), and which is predicted in CDM models for galaxy formation (Nagamine et al. 2004). WGP also found a more significant correlation ( $>3-\sigma$ significance) between $\dot{\psi}_{*}$ and low-ion line width, $\Delta v_{l o w}$. Because $\dot{\psi}_{*}$ is a global quantity integrated over the star forming region of the DLA, such a correlation would imply $\Delta v_{\text {low }}$ is also global, which would indicate $\Delta v_{\text {low }}$ corresponds to the virial velocity of the dark matter halo enclosing the gas. If confirmed, this would be a valuable diagnostic of numerical models for star formation in DLAs, which predict how $\dot{\psi}_{*}$ depends on halo mass (Springel \& Hernquist 2003). At the same time WGP found no evidence for a correlation between $\dot{\psi}_{*}$ and $N(\mathrm{H} \mathrm{I})$ nor $\dot{\psi}_{*}$ and high-ion line width.

WGP tested the $\mathrm{C} \mathrm{II}^{*}$ model, by showing that the observed $l_{c}$ could not be due to radiative excitations from the high-redshift CMB. Nor can it arise from heating by the grain photoelectric effect or C I photoionization if the radiation 
source is the FUV background. Fig. 3 shows the observed $l_{c}$ lies above the predicted cooling rates in all cases. Therefore, in contrast to the Ly $\alpha$ forest, an internal source of heat is required for DLAs. WGB also demonstrated a highly significant correlation between $l_{c}$ and $\kappa$. This is predicted if C II* absorption arises in CNM gas heated by the grain photoelectric effect for a given SFR. It is not naturally predicted for heating by cosmic rays or soft X-rays.

More recently, we tested the CNM model by searching for evidence of Si II* 1264.3 absorption. Because this transition arises from a ${ }^{2} \mathrm{P}_{3 / 2}$ state 410 $\mathrm{K}$ above the ground state, it can only be detected in WNM gas where $T>>400$ $\mathrm{K}$. If $\mathrm{C} \mathrm{II}^{*}$ and Si $\mathrm{II}^{*}$ absorption arise in WNM gas, the optical depth ratio $\tau(\mathrm{Si} \mathrm{II}) / \tau\left(\mathrm{C} \mathrm{II}^{*}\right)$ reaches a maximum value of 0.025 . Howk et al. (2005) find this ratio is significantly smaller than 0.025 and that $T<400 \mathrm{~K}$ for a high- $z$ DLA. This test is independent of the heating mechanism, and only depends on collision strengths and radiative recombination coefficients for the $\mathrm{C} \mathrm{II}^{*}$ and $\mathrm{Si}$ II $^{*}$ transitions. It provides unambiguous evidence for CNM gas in a high- $z$ DLA.

Acknowledgments. The author wishes to thank his collaborators J. X. Prochaska, E. Gawiser, and J. C. Howk for their contributions to this research which is supported by NSF grants AST 0071257 and AST0307824.

\section{References}

Bakes, E. L. O. \& Tielens, A. G. G. M. 1994, ApJ, 427, 822

Garnett, D. R., Shields, G. A., Skillman, E. D., Sagan, S. P., \& Dufour, R. J., 1997, ApJ, 489, 63

Howk, J. C., Wolfe, A. M., \& Prochaska, J. X. 2005, ApJ, 622, 81

Hauser, M. G., \& Dwek, E. 2001, ARA\&A, 39, 249

Nagamine, K., Springel, V., \& Hernquist, L. 2004, MNRAS, 348, 435 (astro-ph/0305409) Peebles, P. J. E. 1993, Principles of Physical Cosmology, Princeton: Princeton University Press, p. 562

Pei, Y. C., Fall, S. M., \& Hauser, M. G. 1999, ApJ, 522, 604

Pettini, M., Smith, L. J., Hunstead, R. W., \& King, D. L. 1994, ApJ, 426, 79

Pottasch, S. R., Wesselius, P. R., \& van Duinen, R. J. 1979, A\&A, 77, 189

Somerville, R. S., Primack, J. R. \& Faber, S. M. 2001, MNRAS, 320, 540

Springel, V. \& Hernquist, L. 2003, MNRAS, 339, 312

Steidel, C.C., Adelberger, K. L., Giavalisco, M., Dickinson, M., \& Pettini, M. 1999, ApJ, 519, 1

Storrie-Lombardi, L. J., \& Wolfe, A. M. 2000, ApJ, 543, 552

Wolfe, A. M., Prochaska, J. X., \& Gawiser, E. 2003, ApJ, 593, 215 (WPG)

Wolfe, A. M., Gawiser, E., \& Prochaska, J. X. 2003, ApJ, 593, 125 (WGP)

Wolfire, M. G., Hollenbach, D., McKee, C. F., Tielens, A. G. G. M., \& Bakes, E. L. O. 1995, ApJ, 443, 152

Wyse, R. F. G., Gilmore, G., \& Franx, M. 1997, ARA\&A, 35, 637 